\title{
Albumin-to-Globulin Ratio at 1 Year after Anti-Tumor Necrosis Factor $\alpha$ Therapy Can Serve as a Prognostic Biomarker in Pediatric Crohn's Disease Patients
}

\author{
Eun Sil Kim, Yiyoung Kwon, Yon Ho Choe, and Mi Jin Kim \\ Department of Pediatrics, Samsung Medical Center, Sungkyunkwan University School of Medicine, Seoul, Korea
}

\author{
Article Info \\ Received October 26, 2020 \\ Revised February 2, 2021 \\ Accepted February 26, 2021 \\ Published online June 9, 2021
}

\section{Corresponding Author}

Yon Ho Choe

ORCID https://orcid.org/0000-0003-1525-7688

E-mail 1101016@skku.edu

Mi Jin Kim

ORCID https://orcid.org/0000-0002-4505-4083

E-mail mijin1217.kim@samsung.com
Background/Aims: The efficacy of biologics for the treatment of Crohn's disease (CD) is affected by the drug concentrations. We aimed to evaluate the importance of albumin and globulin which are known to be associated with drug concentrations as prognostic biomarkers in CD.

Methods: In total, 121 pediatric patients with CD who had received anti-tumor necrosis factor (TNF)- $\alpha$ therapy were retrospectively examined between January 2010 and February 2019.

Results: Relapse was observed in $48.8 \%$ of patients (59/121). The level of calprotectin (odds ratio, 2.13; $p=0.03$ ) and the albumin-to-globulin ratio (AGR) at 1 year after anti-TNF- $\alpha$ therapy (odds ratio, 0.0002; $p=0.003$ ) were associated with relapse. The AGR at 1 year after anti-TNF- $\alpha$ therapy was the only factor associated with the time-to-relapse (hazard ratio, $0.02 ; p<0.001$ ). The optimal AGR cutoff value for the prediction of relapse was 1.47 (area under the curve, 0.916; $p<0.001)$. The median infliximab trough level $(T L)$ was lower in patients with $A G R s<1.47$ than in those with $A G R s \geq 1.47$. Anti-drug antibody (ADA) concentrations were negatively correlated with the AGR at 1 year of anti-TNF- $\alpha$ therapy $(r=-0.413, p=0.032)$.

Conclusions: AGR can be used to predict relapse. Patients with AGRs $<1.47$ at 1 year after anti-TNF- $\alpha$ therapy are more likely to have low drug TLs and develop ADAs, which increase the possibility of relapse than those with $A G R s \geq 1.47$. Therefore, if the AGR at 1 year after anti-TNF- $\alpha$ therapy is less than 1.47, clinicians should monitor disease activity, assess the TLs of the antiTNF- $\alpha$ agents, test for ADAs and determine the appropriate therapeutic strategies. (Gut Liver 2022;16:71-80)

Key Words: Albumin-to-globulin ratio; Relapse; Crohn disease; Pediatric

\section{INTRODUCTION}

Crohn's disease $(\mathrm{CD})$ is a type of inflammatory bowel disease (IBD) that causes chronic inflammation of entire gastrointestinal tract. ${ }^{1}$ The exact cause of $\mathrm{CD}$ remains unknown. However, the currently accepted hypotheses for IBD emphasized the interaction of heredity, environment, and impaired immune system. ${ }^{1,2}$ The disease course of $\mathrm{CD}$ is usually progressive and characterized by periods of clinical remission and relapse. ${ }^{3}$ It is generally known that pediatric-onset $\mathrm{CD}$ is different from that in adults because there is the possibility of linear growth impairment, delayed puberty and the disease is more progressive, extensive, and complicated. ${ }^{4,5}$
Recent studies conducted in adult patients with CD have been reported that factors related to relapse at diagnosis are young diagnosis age, upper gastrointestinal or perianal involvement and stricturing or penetrating disease behavior. $^{6-8}$ There is paucity in the literature on predicting factors related to relapse in pediatric patients with $\mathrm{CD}$. One study reported that there are no significant positive risk factors associated with relapse and another reported low body mass index at diagnosis is related to increased risk of relapse. ${ }^{9,10}$ In contrast, other literature reported that early disease-course factors, such as response to treatment, are more important in predicting early relapse in pediatric patients with $\mathrm{CD}$ than disease activity or disease phenotype at diagnosis. ${ }^{11}$ 
Efficacy of treatment, especially anti-tumor necrosis factor (TNF) $\alpha$ in patients with CD, is affected by serum drug concentrations. Serum drug concentration is influenced by dosage according to body weight, ${ }^{12}$ degradation of drug, ${ }^{13}$ anti-drug antibodies (ADA) to anti-TNF- $\alpha,{ }^{14}$ or drug leakage into gastrointestinal tract. ${ }^{15,16}$ Anti-TNF- $\alpha$ administered to a patient can induce an immune reaction such as the development of ADA, which is associated with low drug trough levels (TLs) and can mediate loss of clinical response to the drug. ${ }^{14,17}$ Albumin is not only used to reflect nutritional status and systemic inflammatory response of patients, ${ }^{18}$ but also used to protect from the catabolism-based degradation of drugs, such as antiTNF- $\alpha .{ }^{19}$ Globulin reflects not as well as inflammation but also immune response to antigenic stimulation such as immunoglobulin formation.

The hypothesis of the study is that albumin and globulin which are related to anti-TNF- $\alpha$ TLs and ADA formation will be associated with relapse in pediatric patients with $\mathrm{CD}$ on maintenance anti-TNF- $\alpha$ therapy. We aimed to evaluate the importance of albumin and globulin as prognostic biomarkers in patients with CD to anti-TNF- $\alpha$ therapy.

\section{MATERIALS AND METHODS}

\section{Patients}

This study was designed as a retrospective observational study conducted at the Department of Pediatrics, Samsung Medical Center between January 2010 and February 2019. Eligible patients had active $\mathrm{CD}$ and were under 18 years of age at the time of diagnosis and first treatment with anti-TNF- $\alpha$. Patients diagnosed as ulcerative colitis, IBDunspecified, primary nonresponders to anti-TNF- $\alpha$, diagnosed ages of more than 18 years old or those with missing data on baseline clinical demographics were excluded. In addition, the subjects had received scheduled anti-TNF- $\alpha$ for at least 1 year and maintained clinical remission for at least 3 months from induction therapy of anti-TNF- $\alpha$ to clearly distinguish relapse from uncontrolled disease activity sustained from diagnosis. CD was diagnosed in accordance with the revised Porto criteria of the European Society for Pediatric Gastroenterology, Hepatology and Nutrition ${ }^{20}$ and disease phenotype classification was based on the Paris classification. ${ }^{21}$

Anti-TNF- $\alpha$ included both infliximab and adalimumab. Infliximab was administered as an induction regimen of $5 \mathrm{mg} / \mathrm{kg}$ at 0,2 and 6 weeks and scheduled maintenance with infliximab was repeated every 8 weeks. Adalimumab was administered subcutaneously on a schedule according to the weight of the patients during induction therapy: 160 $\mathrm{mg}$ at week 0 followed by $80 \mathrm{mg}$ at week 2 for patients over $40 \mathrm{~kg}$ and $80 \mathrm{mg}$ at week 0 followed by $40 \mathrm{mg}$ at week 2 for under $40 \mathrm{~kg}$. After induction treatment, patients $\geq 40 \mathrm{~kg}$ received $40 \mathrm{mg}$ and patients $<40 \mathrm{~kg}$ received $20 \mathrm{mg}$ every other week via subcutaneous injection. Disease activity was evaluated at week 14 from infliximab or adalimumab initiation based on the pediatric Crohn's disease activity index (PCDAI) and patients who showed primary nonresponse were excluded from the analysis. Azathioprine was given at doses of $0.5-1 \mathrm{mg} / \mathrm{kg} /$ day and was later modified when required according to thiopurine methyltransferase genotype and thiopurine metabolite levels of 6-thioguanine nucleotide. Mesalazine was given concomitantly with azathioprine at a dose of $50 \mathrm{mg} / \mathrm{kg} / \mathrm{day}$. This study was approved by the Institutional Review Board of Samsung Medical Center (IRB number: 2020-06-173) and was conducted in accordance with the Declaration of Helsinki. Written informed consent was obtained.

\section{Data collection}

Baseline demographic and clinical data at diagnosis, including sex, age, disease phenotype, growth indicators, previous history of gastrointestinal tract surgery and family history of IBD, laboratory results were collected from electronic medical records. Clinical data at the time of 1 year after anti-TNF- $\alpha$ started were collected retrospectively from electronic charts including the PCDAI score, white blood cell count, hematocrit, platelet count, serum albumin level, serum globulin level, albumin-to-globulin ratio (AGR), erythrocyte sedimentation rate, C-reactive protein, TLs of anti-TNF- $\alpha$, presence of ADA. ADA was quantified using enzyme-linked immunosorbent assay kits (Matriks Biotek, Ankara, Turkey) at an optical density of $450 \mathrm{~nm}$ and TLs of anti-TNF- $\alpha$ were assessed using IDKmonitor ${ }^{\circledR}$ infliximab drug level enzyme-linked immunosorbent assay (Immundiagnostik AG, Bensheim, Germany) for infliximab and SHIKARI ${ }^{\circledR}$ Q-ADA (Matriks Biotek) for adalimumab prior to application of anti-TNF- $\alpha$ administration. The primary endpoint was symptomatic relapse defined as a PCDAI score more than 10 points with a change of at least 10 points from the previous visit, and the need for treatment intensification, which means (1) the addition of a new medication, (2) dose escalation of maintenance treatment or (3) intestinal surgery because of stricturing or penetrating $\mathrm{CD}$.

\section{Statistical analysis}

For statistical comparison between groups according to relapse, the Student $\mathrm{t}$-test or the Wilcoxon rank-sum test was used for continuous variables and the chi-square test or the Fisher exact test was used for categorical variables. 
Univariate and multivariate logistic regression analyses were conducted to examine the association between relapse of $\mathrm{CD}$ and the variables. A univariate analysis was performed to investigate the crucial odds ratio for each variable. Factors with $\mathrm{p}$-value less than 0.1 in the univariate analysis were included in the multivariate analysis using a stepwise selection procedure. Additionally, receiver operating characteristic (ROC) curve was constructed to derive the most accurate cutoff value for continuous variable related to relapse. Factors associated with time to relapse were investigated using univariate and multivariate Cox proportional hazards regression analyses. Factors with pvalue less than 0.1 in the univariate analysis were included in the multivariate analysis. The results are presented as hazard ratios with $95 \%$ confidence interval (CI). The Kaplan-Meier analysis was used to calculate the relapse-free survival and the log-rank test was used to detect overall statistical difference in estimates. In addition, the Pearson correlation coefficient was used to analyze the correlation between variables associated with relapse and ADA concentrations. A p-value of less than 0.05 was considered statistically significant. Statistical analyses were performed using Rex version 3.0.3 (RexSoft Inc., Seoul, Korea).

\section{RESULTS}

\section{Baseline characteristics of patients with and without relapse}

During the study period from January 2010 and February 2019, a total of 121 pediatric CD patients had been treated with anti-TNF- $\alpha$ for at least 1 year and were eligible for analysis. Baseline characteristics for our cohort are described in Table 1.

Among the patients, 62 patients (51.2\%) maintained clinical remission and 59 patients $(48.8 \%)$ experienced relapse at the end of the observation period. The median age of the patients at diagnosis was 14.5 years and 96 of the patients $(79.3 \%)$ were male. Six patients $(5.0 \%)$ had previous bowel resection history before diagnosis and four

Table 1. Comparison between Pediatric Patients with Crohn's Disease Stratified by Relapse Status

\begin{tabular}{|c|c|c|c|c|}
\hline Variable & $\begin{array}{c}\text { Total } \\
(n=121)\end{array}$ & $\begin{array}{l}\text { Remission group } \\
\qquad(\mathrm{n}=62)\end{array}$ & $\begin{array}{l}\text { Relapse group } \\
\qquad(n=59)\end{array}$ & $\mathrm{p}$-value \\
\hline Observational period, yr & $3.7(2.3-6.4)$ & $3.2(1.9-5.0)$ & $4.2(2.4-6.8)$ & 0.060 \\
\hline Age at diagnosis, yr & $14.5(12.6-16.1)$ & $14.4(12.9-16.2)$ & $15.0(12.4-16.0)$ & 0.979 \\
\hline Male sex & 96 (79.3) & 46 (74.2) & 50 (84.8) & 0.227 \\
\hline First-degree family history of IBD & 4 (3.3) & 2 (3.2) & $2(3.4)$ & $>0.999$ \\
\hline Previous bowel resection history & $6(5.0)$ & $2(3.2)$ & $4(6.8)$ & 0.432 \\
\hline Initial BMI, kg/m² & $17.5(15.7-20.5)$ & $17.4(15.6-20.2)$ & $17.6(15.8-20.7)$ & 0.803 \\
\hline Initial PCDAl & $35.0(30.0-40.0)$ & $36.3(30.0-40.0)$ & $35.0(30.0-42.5)$ & 0.799 \\
\hline \multicolumn{5}{|l|}{ Disease location } \\
\hline Ileal (L1) & $12(9.9)$ & $4(6.5)$ & $8(13.5)$ & 0.232 \\
\hline Colonic (L2) & $15(12.4)$ & $6(9.7)$ & 9 (15.3) & \\
\hline |leocolonic (L3) & 94 (77.7) & $52(83.9)$ & $42(71.2)$ & \\
\hline \multicolumn{5}{|l|}{ UGI involvement } \\
\hline None & $40(33.1)$ & $21(33.9)$ & 19 (32.2) & 0.893 \\
\hline Proximal to the ligament of Treitz (L4a) & $19(15.7)$ & $11(17.7)$ & $8(13.6)$ & \\
\hline Distal to the ligament of Treitz and proximal to the distal 1/3 ileum (L4b) & $28(23.1)$ & $14(22.6)$ & $14(23.7)$ & \\
\hline Both (L4ab) & $34(28.1)$ & $16(25.8)$ & $18(30.5)$ & \\
\hline \multicolumn{5}{|l|}{ Luminal disease behavior } \\
\hline Non-stricturing\&non-penetrating (B1) & 105 (86.8) & 56 (90.3) & $49(83.1)$ & 0.432 \\
\hline Stricturing (B2) & $12(9.9)$ & $5(8.1)$ & 7 (11.9) & \\
\hline Penetrating (B3) & 4 (3.3) & $1(1.6)$ & $3(5.1)$ & \\
\hline Perianal disease & 79 (65.3) & $42(67.7)$ & $37(62.7)$ & 0.697 \\
\hline Growth retardation & $21(17.4)$ & 7 (11.3) & $14(23.7)$ & 0.117 \\
\hline Steroid & $21(17.4)$ & $10(16.1)$ & $11(18.6)$ & 0.901 \\
\hline Azathioprine & 110 (90.9) & $56(90.3)$ & 54 (91.5) & $>0.999$ \\
\hline Mesalazine & $115(95.0)$ & $60(96.8)$ & 55 (93.2) & 0.432 \\
\hline \multicolumn{5}{|l|}{ Anti-TNF-a } \\
\hline Infliximab & 97 (80.2) & 47 (75.8) & 50 (84.8) & 0.432 \\
\hline Adalimumab & $24(19.8)$ & 15 (24.2) & 9 (15.3) & 0.315 \\
\hline Age at anti-TNF-a start, yr & $15.0 \pm 3.1$ & $15.1 \pm 3.2$ & $14.8 \pm 2.9$ & 0.547 \\
\hline
\end{tabular}

Data are presented as median (IQR), number (\%), or mean \pm SD.

$I Q R$, interquartile range; IBD, inflammatory bowel disease; BMI, body mass index; PCDAl, pediatric Crohn's disease activity index; UGI, upper gastrointestinal; TNF, tumor necrosis factor. 
patients (3.3\%) had first-degree family history of IBD. The median observational duration was 3.7 years. Ninety-seven patients $(80.2 \%)$ were treated with infliximab and $24 \mathrm{pa}$ tients (19.8\%) with adalimumab. There were no significant differences in disease phenotype, concomitant medication (azathioprine, mesalazine), type of anti-TNF- $\alpha$, age at antiTNF- $\alpha$ therapy start.

Baseline characteristics at 1 year after anti-TNF- $\alpha$ treatment are shown in Table 2. When comparing patients between remission group and relapse group at the end of the study period, globulin and AGR at 1 year after anti-TNF- $\alpha$ treatment were factors showing a statistically valid difference between two groups ( $\mathrm{p}<0.001$, respectively).
Other baseline characteristics and comparisons between patients who did and did not relapse at diagnosis and at 1 year after anti-TNF- $\alpha$ treatment are presented in Tables 1 and 2.

\section{Factors associated with relapse}

According to the univariate logistic regression analysis, there were significant associations between hematocrit, Creactive protein, globulin, AGR at diagnosis and calprotectin, globulin and AGR at 1 year after anti-TNF- $\alpha$ therapy, and relapse (Table 3). These variables were included in the multivariate analysis and revealed that calprotectin (odds ratio, 2.13; 95\% CI, 1.64 to $2.91 ; \mathrm{p}=0.03$ ) and $\mathrm{AGR}$ at 1

Table 2. Comparison of Baseline Characteristics after 1 Year of Anti-Tumor Necrosis Factor a Treatment

\begin{tabular}{lcccc}
\hline \multicolumn{1}{c}{ Characteristics } & Total $(\mathrm{n}=121)$ & Remission group $(\mathrm{n}=62)$ & Relapse group $(\mathrm{n}=59)$ & $\mathrm{p}$-value \\
\hline PCDAl & $0(0-5)$ & $0(0-5)$ & $0(0-5)$ & 0.979 \\
WBC count, $\times 10^{3} / \mu \mathrm{L}$ & $5.3(4.5-6.7)$ & $5.8(4.7-6.9)$ & $5.4(4.5-6.7)$ & 0.182 \\
Hematocrit, $\%$ & $39.1(36.2-41.9)$ & $40.0(37.3-42.8)$ & $37.7(35.8-40.9)$ & 0.053 \\
Platelet count, $\times 10^{3} / \mu \mathrm{L}$ & $275.0(233.8-317.3)$ & $284.0(240.0-318.0)$ & $273.0(225.0-309.5)$ & 0.515 \\
ESR, $\mathrm{mm} / \mathrm{hr}$ & $6.0(2.0-13.3)$ & $4.0(2.0-13.0)$ & $7.0(3.0-14.0)$ & 0.110 \\
CRP, $\mathrm{mg} / \mathrm{dL}$ & $0.03(0.03-0.06)$ & $0.03(0.03-0.03)$ & $0.03(0.03-0.08)$ & 0.070 \\
Calprotectin, $\mathrm{mg} / \mathrm{mL}$ & $105.5(34.9-851.7)$ & $93.3(37.0-876.8)$ & $115.3(23.7-835.3)$ & 0.381 \\
Albumin, $\mathrm{g} / \mathrm{dL}$ & $4.4(4.1-4.6)$ & $4.4(4.2-4.6)$ & $4.3(4.1-4.5)$ & 0.143 \\
Globulin, g/dL & $2.8(2.5-3.2)$ & $2.6(2.3-2.8)$ & $3.2(2.8-3.7)$ & $<0.001$ \\
AGR & $1.53 \pm 0.28$ & $1.70 \pm 0.23$ & $1.36 \pm 0.21$ & $<0.001$ \\
\hline
\end{tabular}

Data are presented as median (IQR) or mean \pm SD.

IQR, interquartile range; PCDAl, pediatric Crohn's disease activity index; WBC, white blood cell; ESR, erythrocyte sedimentation rate; CRP, Creactive protein; $A G R$, albumin-to-globulin ratio.

Table 3. Logistic Regression Analyses of Factors Associated with Relapse in Patients with Crohn's Disease

\begin{tabular}{|c|c|c|c|c|c|c|}
\hline \multirow[t]{2}{*}{ Factor } & \multicolumn{3}{|c|}{ Univariate logistic regression $(n=121)$} & \multicolumn{3}{|c|}{$\begin{array}{l}\text { Multivariate logistic regression with } \\
\text { stepwise selection }(n=121)\end{array}$} \\
\hline & $\mathrm{OR}$ & $95 \% \mathrm{Cl}$ & p-value & Adjusted OR & $95 \% \mathrm{Cl}$ & p-value \\
\hline Sex (female vs male) & 1.93 & $0.78-4.80$ & 0.156 & & & \\
\hline Age at diagnosis & 0.98 & $0.86-1.12$ & 0.777 & & & \\
\hline First-degree family history of IBD & 1.05 & $0.14-7.73$ & 0.960 & & & \\
\hline $\mathrm{BMI}$ at diagnosis & 1.00 & $0.90-1.10$ & 0.935 & & & \\
\hline PCDAl at diagnosis & 1.00 & $0.97-1.03$ & 0.915 & & & \\
\hline WBC count at diagnosis & 0.93 & $0.83-1.04$ & 0.191 & & & \\
\hline Hematocrit at diagnosis & 0.91 & $0.83-0.99$ & 0.028 & 0.95 & $0.81-1.11$ & 0.515 \\
\hline Platelet count at diagnosis & 1.00 & $0.99-1.00$ & 0.476 & & & \\
\hline Erythrocyte sedimentation rate at diagnosis & 1.01 & $0.99-1.03$ & 0.397 & & & \\
\hline C-reactive protein at diagnosis & 1.12 & $1.00-1.26$ & 0.060 & 1.09 & $0.90-1.32$ & 0.400 \\
\hline Calprotectin at diagnosis & 1.07 & $0.14-22.84$ & 0.996 & & & \\
\hline Albumin at diagnosis & 0.17 & $0.02-1.90$ & 0.150 & & & \\
\hline Globulin at diagnosis & 2.27 & $1.05-4.93$ & 0.038 & 0.35 & $0.04-2.93$ & 0.329 \\
\hline AGR at diagnosis & 0.29 & $0.09-0.95$ & 0.041 & 1.43 & $0.06-34.2$ & 0.824 \\
\hline BMI at 1 year after anti-TNF-a therapy & 1.05 & $0.73-1.51$ & 0.792 & & & \\
\hline Calprotectin at 1 year after anti-TNF-a therapy & 2.45 & $1.85-2.73$ & 0.040 & 2.13 & $1.64-2.91$ & 0.030 \\
\hline Albumin at 1 year after anti-TNF-a therapy & 0.38 & $0.13-1.15$ & 0.088 & & & \\
\hline Globulin at 1 year after anti-TNF-a therapy & 43.60 & $10.70-177.60$ & $<0.001$ & 5.78 & $0.52-63.87$ & 0.153 \\
\hline AGR at 1 year after anti-TNF-a therapy & 0.0035 & $0.0004-0.0313$ & $<0.001$ & 0.0002 & $9.40 \times 10^{-7}-0.06$ & 0.003 \\
\hline
\end{tabular}

$\mathrm{OR}$, odds ratio; $\mathrm{Cl}$, confidence interval; IBD, inflammatory bowel disease; BMI, body mass index; PCDAl, pediatric Crohn's disease activity index; WBC, white blood cell; AGR, albumin-to-globulin ratio; TNF, tumor necrosis factor. 
year after anti-TNF- $\alpha$ therapy (odds ratio, 0.0002; 95\% CI, $9.40 \times 10^{-7}$ to $0.06 ; \mathrm{p}=0.003$ ) were factors significantly associated with relapse.

According to the ROC curve shown in Fig. 1, the most accurate cutoff value for AGR at 1 year after anti-TNF- $\alpha$ therapy associated with relapse was 1.47 (area under the ROC curve 0.916 , 95\% CI 0.870 to 0.961 , sensitivity $84.7 \%$, specificity $90.3 \%$, positive predictive value $89.3 \%$, negative predictive value $86.2 \%, \mathrm{p}<0.001$ ).

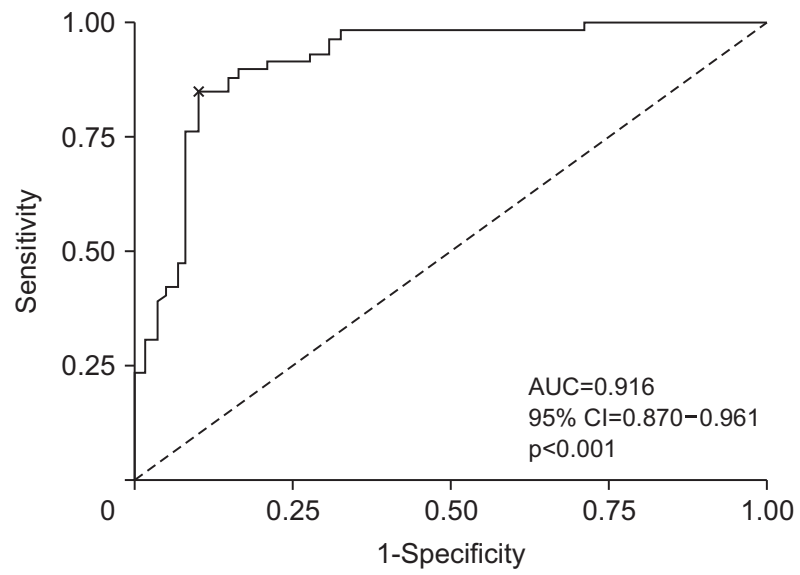

Fig. 1. Receiver operator characteristic curve for the prediction of relapse based on the albumin-to-globulin ratio at 1 year after anti-tumor necrosis factor a therapy.

AUC, area under the receiver operating characteristic curve; $\mathrm{Cl}$, confidence interval.

\section{Factors associated with time to relapse}

According to the univariate Cox proportional hazards regression analysis, platelet count at diagnosis and globulin at 1 year after anti-TNF- $\alpha$ therapy showed positive association with time to relapse, while AGR after 1 year of anti-TNF- $\alpha$ therapy showed negative association with time to relapse (Table 4). Factors that showed p-value less than 0.1 on univariate analysis, platelet count and erythrocyte sedimentation rate at diagnosis, globulin and AGR after 1 year of anti-TNF- $\alpha$ therapy were included in the multivariate analysis. According to the multivariate Cox proportional hazards regression analysis, only AGR at 1 year of anti-TNF- $\alpha$ therapy was significantly associated with time to relapse (hazard ratio, $0.02 ; 95 \% \mathrm{CI}, 0.002$ to 0.181 ; $\mathrm{p}<0.001)$.

To evaluate relapse-free survival in relationship to AGR at 1 year of anti-TNF- $\alpha$ therapy, the Kaplan-Meier survival curves were calculated using cutoff value of AGR as 1.47. Relapse-free survival curve showed a significant trend with a poorer outcome in patients with AGR $<1.47$ compared to those with $\geq 1.47$ (log-rank test $p=0.016$ ) (Fig. 2).

\section{Serum TLs and ADA to anti-TNF- $\alpha$}

The median infliximab TLs at 1 year of infliximab treatment were significantly lower in patients with AGR $<1.47$ than in those with AGR $\geq 1.47(2.6 \mu \mathrm{g} / \mathrm{mL}$ vs $4.7 \mu \mathrm{g} / \mathrm{mL}$, $\mathrm{p}=0.028$ ) (Fig. 3A). In patients treated with adalimumab, patients with AGR $<1.47$ tended to have lower TLs than

Table 4. Cox Proportional Hazard Regression Analysis of Factors Associated with Time to Relapse in Patients with Crohn's Disease

\begin{tabular}{|c|c|c|c|c|c|c|}
\hline \multirow{2}{*}{ Factor } & \multicolumn{3}{|c|}{ Univariate Cox analysis } & \multicolumn{3}{|c|}{ Multivariate analysis with stepwise selection $(n=121)$} \\
\hline & $\mathrm{HR}$ & $95 \% \mathrm{Cl}$ & $p$-value & HR & $95 \% \mathrm{Cl}$ & p-value \\
\hline Sex (female vs male) & 1.06 & $0.51-2.17$ & 0.881 & & & \\
\hline Age at diagnosis & 1.04 & $0.96-1.13$ & 0.351 & & & \\
\hline First-degree family history of IBD & 1.16 & $0.28-4.81$ & 0.834 & & & \\
\hline $\mathrm{BMI}$ at diagnosis & 1.06 & $0.99-1.13$ & 0.106 & & & \\
\hline PCDAl at diagnosis & 1.01 & $0.99-1.04$ & 0.227 & & & \\
\hline WBC count at diagnosis & 0.98 & $0.90-1.06$ & 0.555 & & & \\
\hline Hematocrit at diagnosis & 0.98 & $0.93-1.03$ & 0.416 & & & \\
\hline Platelet count at diagnosis & 1.01 & $1.00-1.01$ & 0.005 & 1.00 & $1.00-1.01$ & 0.062 \\
\hline Erythrocyte sedimentation rate at diagnosis & 1.01 & $1.00-1.01$ & 0.080 & 0.99 & $0.98-1.00$ & 0.179 \\
\hline C-reactive protein at diagnosis & 1.05 & $0.97-1.14$ & 0.226 & & & \\
\hline Calprotectin at diagnosis & 1.04 & $0.73-1.45$ & 0.998 & & & \\
\hline Albumin at diagnosis & 0.86 & $0.55-1.37$ & 0.530 & & & \\
\hline Globulin at diagnosis & 1.45 & $0.81-2.59$ & 0.210 & & & \\
\hline AGR at diagnosis & 0.67 & $0.28-1.62$ & 0.377 & & & \\
\hline BMI at 1 year of anti-TNF-a therapy & 1.05 & $0.69-1.60$ & 0.819 & & & \\
\hline Calprotectin at 1 year of anti-TNF-a therapy & 1.06 & $0.56-1.37$ & 0.895 & & & \\
\hline Albumin at 1 year of anti-TNF-a therapy & 0.76 & $0.41-1.43$ & 0.402 & & & \\
\hline Globulin at 1 year of anti-TNF-a therapy & 2.68 & $1.72-4.18$ & $<0.001$ & 0.87 & $0.37-2.02$ & 0.742 \\
\hline AGR at 1 year of anti-TNF-a therapy & 0.04 & $0.01-0.13$ & $<0.001$ & 0.02 & $0.002-0.181$ & $<0.001$ \\
\hline
\end{tabular}

$\mathrm{HR}$, hazard ratio; $\mathrm{Cl}$, confidence interval; IBD, inflammatory bowel disease; BMI, body mass index; PCDAl, pediatric Crohn's disease activity index; WBC, white blood cell; AGR, albumin-to-globulin ratio; TNF, tumor necrosis factor. 
those with AGR $\geq 1.47$, however these were not statistically significant $(7.0 \mu \mathrm{g} / \mathrm{mL}$ vs $10.6 \mu \mathrm{g} / \mathrm{mL}, \mathrm{p}=0.06)$ (Fig. 3B).

Analyzing association of ADA concentrations and AGR at 1 year after anti-TNF- $\alpha$ therapy, ADA concentrations at 1 year after anti-TNF- $\alpha$ therapy was negatively correlated with AGR at 1 year of anti-TNF- $\alpha(\mathrm{r}=-0.413, \mathrm{p}=0.032)$.

\section{DISCUSSION}

In this study, we investigated laboratory and treatmentassociated factors potentially associated with relapse in pediatric patients with $\mathrm{CD}$ who had been receiving treatment with anti-TNF- $\alpha$, especially albumin and globulin which are known to be related to drug concentrations. We found a significant association between AGR at 1 year after

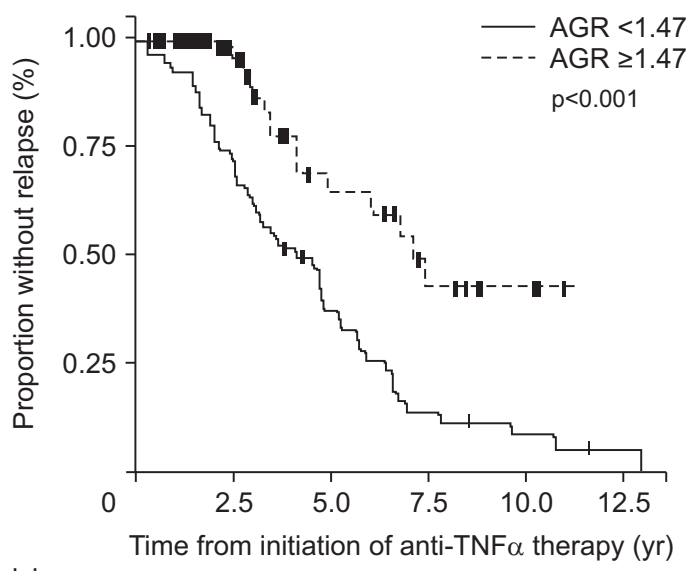

At risk

$\begin{array}{lllllll}- \text { AGR }<1.47 & 67 & 37 & 14 & 7 & 3 & 0 \\ ---A G R \geq 1.47 & 54 & 36 & 16 & 6 & 3 & 1\end{array}$

Fig. 2. Relapse-free survival curve according to the albumin-toglobulin ratio (AGR) at 1 year after anti-tumor necrosis factor (TNF) a therapy.

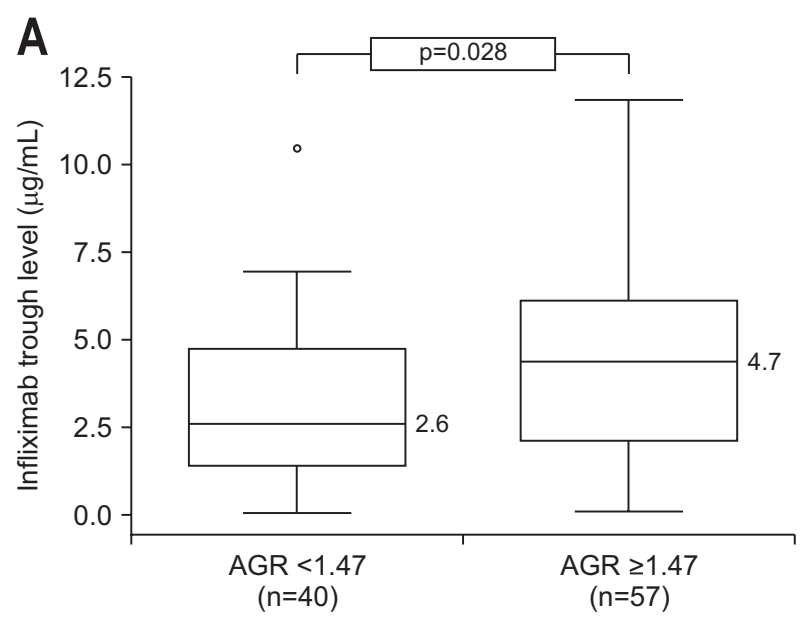

anti-TNF- $\alpha$ treatment and relapse, and derived an optimal AGR cutoff value of 1.47 that could best stratify patients with and without relapse. To the best of our knowledge, this is the first study on predictors associated with relapse during anti-TNF- $\alpha$ therapy in pediatric patients with CD except for calprotectin. ${ }^{22}$

Despite an effective treatment that enables remission to be reached, a degree of subclinical inflammation may remain within the gastrointestinal tract, contributing to a risk of clinical relapse in some patients with $\mathrm{CD} .{ }^{6,8}$ Unfortunately, it is difficult to predict if there will be a relapse and when it will occur. Zubin and Peter ${ }^{23}$ reported that only fecal calprotectin correlates with endoscopic activity after treatment. However, fecal calprotectin is not easy to collect samples from pediatric patients and it takes several days to confirm the results in real world setting. Therefore, defining factors to predict relapse is required to determine appropriate therapeutic strategies, because there is a limitation in evaluating the treatment response and deciding immediately stratified treatment of risk group in the outpatient clinic.

To date, most studies predict the prognosis of patients according to factors at diagnosis in adult or pediatric CD. ${ }^{6,7,9,10,24,25}$ We reported that an AGR of 1.47 at 1 year after anti-TNF- $\alpha$ therapy was predictive of relapse, with a hazard ratio of 0.02 (95\% CI, 0.002 to $0.181 ; \mathrm{p}<0.001$ ) and good predictive accuracy (area under the ROC curve, 0.916). Furthermore, the Kaplan-Meier time to relapse plots using AGR $<1.47$ at 1 year after anti-TNF- $\alpha$ therapy, determined that patients with lower AGR showed increased risk of developing relapse significantly than those with higher AGR values ( $\mathrm{p}=0.016)$. This result demonstrates that AGR at 1 year after anti-TNF- $\alpha$ therapy is a valuable factor for predicting relapse. We did not find an association with C-reactive protein or erythrocyte sedi-

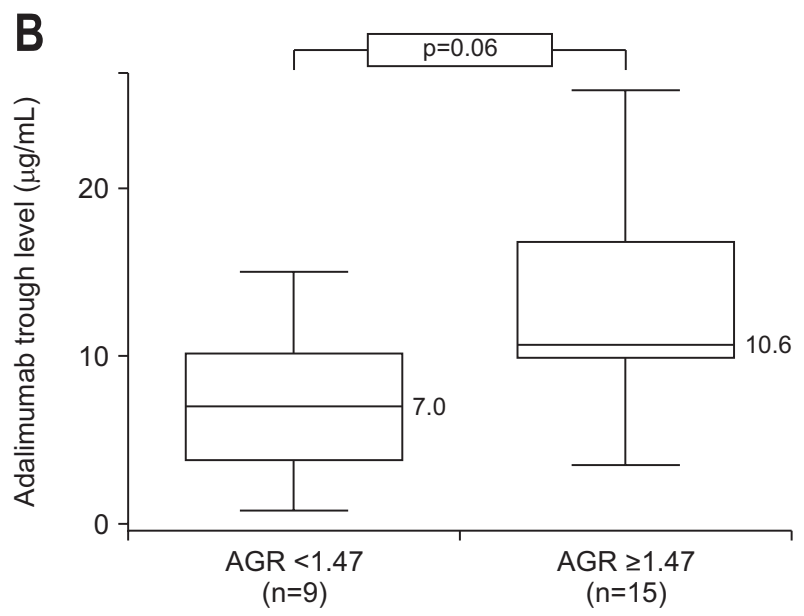

Fig. 3. Anti-TNF-a agent trough levels in patients after 1 year of anti-TNF-a therapy. (A) Infliximab and (B) adalimumab.

TNF, tumor necrosis factor; AGR, albumin-to-globulin ratio. 
mentation rate at 1 year after anti-TNF- $\alpha$ therapy and relapse, which several previous studies evaluated to be useful in predicting relapse in CD patients (Table 2). ${ }^{26-28}$

Albumin and globulin are known as two major components of serum. The serum albumin level is considered to reflect not only the nutritional status but also the inflammatory status of the body which usually maintains a certain amount to achieve homeostasis. ${ }^{29}$ Albumin exhibits protective effects that maintains the function of several compounds by binding to these compounds such as a variety of drugs, hydrophobic organic anion, divalent cations and others more. ${ }^{18}$ In addition, albumin provides antioxidant activity of normal plasma, which means that albumin is important in inflammatory process. ${ }^{30}$ It was reported that patients with low serum albumin diagnosed with IBD are prone to have a higher clearance rate of antiTNF- $\alpha$, have a more severe course of the disease and develop more complications during and after surgical interventions. $^{15,26,31-33}$ The results of our study are in agreement with these results. The median infliximab TLs at 1 year of infliximab treatment were significantly lower in patients with AGR $<1.47$ than in those with AGR $\geq 1.47$ ( $\mathrm{p}=0.028$ ). The same trend was observed with patients treated with adalimumab, but the reason this result was not statistically significant is due to the small number of patients receiving adalimumab $(n=24)$.

On the other hand, globulin is divided into threefraction $(\alpha, \beta$, and $\gamma)$ based on its electrophoretic mobility, and hundreds of different proteins are included. Specific groups of proteins included in the globulins are produced in response to inflammatory stimuli. ${ }^{34}$ These include the acute and chronic phase proteins and the immunoglobulins as the result of increased hepatic synthesis mediated by cytokines. Several laboratory results can be used for diagnosis and monitoring of inflammatory and immune status of the body, and globulin can be one of them. The causes of increased globulin in CD patients treated with anti-TNF- $\alpha$ can be poorly controlled chronic or subclinical inflammation or development of immunogenicity to anti-TNF- $\alpha$ agents. $^{19}$

In our study, the positive rate of ADA within 1 year was $8.3 \%(10 / 121)$ and the positive rate of ADA during observational period was $15.7 \%$ (19/121). The correlation coefficient between ADA concentrations and AGR at 1 year after anti-TNF- $\alpha$ therapy was -0.413 , showing moderate negative correlation. According to previous study conducted by Moses et al., ${ }^{35}$ the median time taking to formation of ADA was 13.2 months in pediatric IBD patients treated with anti-TNF- $\alpha$ therapy, and $90.3 \%$ of subjects showed ADA free survival at 1 year of treatment. Compared to previous study with our study, moderate correlation between ADA formation and AGR at 1 year after anti-TNF- $\alpha$ therapy can result from relatively short observational period and small proportion of patients with positive ADA during study period. Also, considering the majority of patients (110/121, $90.9 \%$ ) treated with combination therapy of azathioprine and anti-TNF- $\alpha$ therapy, the correlation coefficient value of ADA and AGR at 1 year after anti-TNF- $\alpha$ therapy $(r=-0.413)$ might be underestimated. These results are in line with previous research which demonstrated that the use of concomitant immunomodulators such as azathioprine with anti-TNF- $\alpha$ therapy was associated with a lower risk for ADA formation. ${ }^{36,37}$ Considering these points, a long-term study is needed in the future on the relationship between ADA and AGR, and further studies are needed on the relationship between ADA and $\gamma$-globulin, which is associated immunogenicity.

In diseases where chronic inflammation and nutrition are involved in the pathogenesis of diseases, AGR, as combination of albumin and globulin, reflecting nutritional and inflammatory status could play an important role in evaluating patients. Therefore, their importance as noninvasive prognostic factors for various diseases related to inflammation has been emerging. ${ }^{38-44}$ For example, low pretreatment AGR is an independent risk factor for recurrence and progression in various cancer. ${ }^{39-43,45}$ It has been known that there is a reciprocal promoting effect between tumors and inflammation. This means that inflammatory cytokines produced by tumor cells as well as inflammatory factors developed when damaged tissues are remodelled, rehabilitated and vascularized influence cancer-related inflammation. ${ }^{46}$ In addition, malnutrition commonly occurs in cancer patients which contribute to disease progression.

It is hypothesized that the pathogenesis of $\mathrm{CD}$ is a complex interaction between genetic susceptibility, environmental factors and altered gut microbiota, leading to dysregulated innate and adaptive immune response and resulting in chronic inflammation of gastrointestinal tract. ${ }^{3}$ In addition, serum albumin and globulin levels are known to be factors representing nutritional and inflammatory conditions and modifying clearance of monoclonal antibodies such as anti-TNF- $\alpha$ as mentioned above. When our patients treated with infliximab were divided into according to the AGR cutoff value 1.47, the group with lower AGR revealed significant low infliximab TLs than that with higher AGR $(2.6 \mu \mathrm{g} / \mathrm{mL}$ vs $4.7 \mu \mathrm{g} / \mathrm{mL})$. These results are consistent with our results that CD patients with low AGR tend to have high tendency of anti-TNF- $\alpha$ degradation and ADA formation, which means that therapeutic response to anti-TNF- $\alpha$ might be reduced in those patients because of low TLs.

The cutoff value of AGR after 1 year of anti-TNF- $\alpha$ 
therapy (1.47) used to predict the clinical relapse of disease was determined by constructing a ROC curve. The cutoff value for AGR after anti-TNF- $\alpha$ therapy provides better sensitivity and specificity values for predicting relapse than other parameters evaluated in this study. As a result, patients with AGR $<1.47$ at 1 year after anti-TNF- $\alpha$ therapy are more likely to have a stage of enhanced or active mucosal inflammation, which progresses to an eventual clinical relapse of the disease or are prone to the development of immunological reaction against the drug, or both.

These findings may have important implications in clinical practice. This is because AGR can assist to predict the likelihood of maintaining adequate TLs of anti-TNF- $\alpha$ and developing ADA for patients who have reached clinical remission and potentially help predict attaining sustained remission. If AGR is less than 1.47 at 1 year after anti-TNF- $\alpha$ therapy, clinicians should consider checking the TLs of anti-TNF- $\alpha$, ADA and modifying therapeutic strategies such as increasing immunomodulators or dose intensification of anti-TNF- $\alpha$ even in asymptomatic patients.

The strength of this study is that strict definitions were used to include pediatric patients with $\mathrm{CD}$ for at least 3 months in clinical remission, thereby reducing the potential influence of preceding flares on the baseline level of biochemical markers of inflammation. Also, the routinely measured factor in outpatient clinic, AGR, has been suggested as a marker of relapse in patients with CD. In clinical practice, calprotectin is difficult to check due to poor compliance in pediatric patients and results are not available on the day of the examination, so it is often impossible to check the results at the time of the outpatient clinic. In contrast, AGR can be easily used because it is routinely included in laboratory tests performed in the clinic and check the results immediately. Potential advantages offered by this variable in risk stratification include time efficiency, cost-effectiveness, and minimal variability in results when measured in different settings.

The limitations of our study are its retrospective nature and, consequently, unstructured follow-up of patients. However, all patients attended the outpatient clinic at regular intervals after baseline evaluation and the extraction of clinical disease activity from medical records was possible at all outpatient visits using the PCDAI. Also, due to the small number of sample size in remission group $(\mathrm{n}=62)$ and relapse group ( $\mathrm{n}=59)$, sufficient statistical analysis was limited regarding investigation between two groups. For example, there were no statistical difference in the history of bowel resection and disease behavior between remission and relapse group ( $>0.05$ ) as shown in Table 1. Further well-designed prospective studies with larger cohorts are required to address these limitations. In addition, the range of calprotectin that can be measured in our hospital was limited to $0-1,000 \mu \mathrm{g} / \mathrm{g}$. For this reason, the evaluation of quantitative calprotectin as a predictor of relapse and time to relapse was limited in this study.

In conclusion, we found that AGR was a useful surrogate marker for predicting relapse. Patients with AGR $<1.47$ at 1 year after anti-TNF- $\alpha$ therapy are more likely to have low TLs and develop ADA, which increases the possibility of relapse than patients with AGR $\geq 1.47$. Therefore, it is helpful to check the AGR and if AGR at 1 year after anti-TNF- $\alpha$ therapy is below 1.47 , clinicians should monitor the disease activity, check TLs of anti-TNF- $\alpha$, ADA and determine appropriate therapeutic strategies.

\section{CONFLICTS OF INTEREST}

No potential conflict of interest relevant to this article was reported.

\section{ACKNOWLEDGEMENTS}

This work was supported by the National Research Foundation of Korea (NRF) grant funded by the Korea government (MSIT) (grant number: 2020R1A2C2007192).

\section{AUTHOR CONTRIBUTIONS}

Study concept and design: E.S.K. Data acquisition: Y.K. Data analysis and interpretation: E.S.K. Writing- original draft: E.S.K. Writing - review \& editing: E.S.K., M.J.K. Study supervision: M.J.K., Y.H.C. Approval of final manuscript: all authors.

\section{ORCID}

Eun Sil Kim https://orcid.org/0000-0003-2012-9867 Yiyoung Kwon https://orcid.org/0000-0001-5600-2070 Yon Ho Choe https://orcid.org/0000-0003-1525-7688 Mi Jin Kim https://orcid.org/0000-0002-4505-4083

\section{REFERENCES}

1. Baumgart DC, Sandborn WJ. Inflammatory bowel disease: clinical aspects and established and evolving therapies. Lancet 2007;369:1641-1657.

2. Hyams JS. Inflammatory bowel disease. Pediatr Rev 2005; 
26:314-320.

3. Rosen MJ, Dhawan A, Saeed SA. Inflammatory bowel disease in children and adolescents. JAMA Pediatr 2015; 169:1053-1060.

4. Cuffari C. Inflammatory bowel disease in children: a pediatrician's perspective. Minerva Pediatr 2006;58:139-157.

5. Cuffari C, Darbari A. Inflammatory bowel disease in the pediatric and adolescent patient. Gastroenterol Clin North Am 2002;31:275-291.

6. Sahmoud T, Hoctin-Boes G, Modigliani R, et al. Identifying patients with a high risk of relapse in quiescent Crohn's disease. The GETAID Group. The Groupe d'Etudes Thérapeutiques des Affections Inflammatoires Digestives. Gut 1995;37:811-818.

7. Liverani E, Scaioli E, Digby RJ, Bellanova M, Belluzzi A. How to predict clinical relapse in inflammatory bowel disease patients. World J Gastroenterol 2016;22:1017-1033.

8. Yarur AJ, Strobel SG, Deshpande AR, Abreu MT. Predictors of aggressive inflammatory bowel disease. Gastroenterol Hepatol (N Y) 2011;7:652-659.

9. Chauhan N, Khan HH, Kumar S, Lyons H. Clinical variables as predictors of first relapse in pediatric Crohn's disease. $\mathrm{Cu}$ reus 2019;11:e4980.

10. Hojsak I, Pavić AM, Mišak Z, Kolaček S. Risk factors for relapse and surgery rate in children with Crohn's disease. Eur J Pediatr 2014;173:617-621.

11. Ziv-Baran T, Hussey S, Sladek M, et al. Response to treatment is more important than disease severity at diagnosis for prediction of early relapse in new-onset paediatric Crohn's disease. Aliment Pharmacol Ther 2018;48:1242-1250.

12. Scaldaferri F, D'Ambrosio D, Holleran G, et al. Body mass index influences infliximab post-infusion levels and correlates with prospective loss of response to the drug in a cohort of inflammatory bowel disease patients under maintenance therapy with Infliximab. PLoS One 2017;12:e0186575.

13. Hemperly A, Sandborn WJ, Vande Casteele N. Clinical pharmacology in adult and pediatric inflammatory bowel disease. Inflamm Bowel Dis 2018;24:2527-2542.

14. Garcês S, Demengeot J, Benito-Garcia E. The immunogenicity of anti-TNF therapy in immune-mediated inflammatory diseases: a systematic review of the literature with a metaanalysis. Ann Rheum Dis 2013;72:1947-1955.

15. Rosen MJ, Minar P, Vinks AA. Review article: applying pharmacokinetics to optimise dosing of anti-TNF biologics in acute severe ulcerative colitis. Aliment Pharmacol Ther 2015;41:1094-1103.

16. Choi SY, Kang B, Choe YH. Serum infliximab cutoff trough level values for maintaining hematological remission in pediatric inflammatory bowel disease. Gut Liver 2019;13:541548.

17. Nakase H. Optimizing the use of current treatments and emerging therapeutic approaches to achieve therapeutic success in patients with inflammatory bowel disease. Gut Liver 2020;14:7-19.

18. Levitt DG, Levitt MD. Human serum albumin homeostasis: a new look at the roles of synthesis, catabolism, renal and gastrointestinal excretion, and the clinical value of serum albumin measurements. Int J Gen Med 2016;9:229-255.

19. Schoenefuss F, Hoffmann P. Serum $\gamma$-globulin and albumin concentrations predict secondary loss of response to antiTNFa in inflammatory bowel disease patients. Eur J Gastroenterol Hepatol 2019;31:1563-1568.

20. Levine A, Koletzko S, Turner D, et al. ESPGHAN revised porto criteria for the diagnosis of inflammatory bowel disease in children and adolescents. J Pediatr Gastroenterol Nutr 2014;58:795-806.

21. Levine A, Griffiths A, Markowitz J, et al. Pediatric modification of the Montreal classification for inflammatory bowel disease: the Paris classification. Inflamm Bowel Dis 2011;17:1314-1321.

22. Foster AJ, Smyth M, Lakhani A, Jung B, Brant RF, Jacobson K. Consecutive fecal calprotectin measurements for predicting relapse in pediatric Crohn's disease patients. World J Gastroenterol 2019;25:1266-1277.

23. Zubin G, Peter L. Predicting endoscopic Crohn's disease activity before and after induction therapy in children: a comprehensive assessment of PCDAI, CRP, and fecal calprotectin. Inflamm Bowel Dis 2015;21:1386-1391.

24. Tibble JA, Sigthorsson G, Bridger S, Fagerhol MK, Bjarnason I. Surrogate markers of intestinal inflammation are predictive of relapse in patients with inflammatory bowel disease. Gastroenterology 2000;119:15-22.

25. Diederen K, Hoekman DR, Leek A, et al. Raised faecal calprotectin is associated with subsequent symptomatic relapse, in children and adolescents with inflammatory bowel disease in clinical remission. Aliment Pharmacol Ther 2017;45:951960.

26. Qin G, Tu J, Liu L, et al. Serum albumin and C-reactive protein/albumin ratio are useful biomarkers of Crohn's disease activity. Med Sci Monit 2016;22:4393-4400.

27. André C, Descos L, Vignal J, Gillon J. C-reactive protein as a predictor of relapse in asymptomatic patients with Crohn's disease. Scott Med J 1983;28:26-29.

28. Brignola C, Campieri M, Bazzocchi G, Farruggia P, Tragnone A, Lanfranchi GA. A laboratory index for predicting relapse in asymptomatic patients with Crohn's disease. Gastroenterology 1986;91:1490-1494.

29. Don BR, Kaysen G. Serum albumin: relationship to inflammation and nutrition. Semin Dial 2004;17:432-437.

30. Taverna M, Marie AL, Mira JP, Guidet B. Specific antioxidant properties of human serum albumin. Ann Intensive Care 2013;3:4. 
31. Nisar PJ, Appau KA, Remzi FH, Kiran RP. Preoperative hypoalbuminemia is associated with adverse outcomes after ileoanal pouch surgery. Inflamm Bowel Dis 2012;18:10341041.

32. Najarian RM, Ashworth LA, Wang HH, Bousvaros A, Goldsmith JD. Microscopic/"backwash" ileitis and its association with Colonic disease in new onset pediatric ulcerative colitis. J Pediatr Gastroenterol Nutr 2019;68:835-840.

33. Huang W, Tang Y, Nong L, Sun Y. Risk factors for postoperative intra-abdominal septic complications after surgery in Crohn's disease: a meta-analysis of observational studies. J Crohns Colitis 2015;9:293-301.

34. Tomasi TB Jr. Human gamma globulin. Blood 1965;25:382403.

35. Moses J, Lambert-Jenkins K, Momotaz H, et al. Time to antibody detection and associated factors for presence of antidrug antibodies in pediatric inflammatory bowel disease patients treated with anti-TNF therapy. Eur J Gastroenterol Hepatol 2019;31:1228-1233.

36. Vermeire S, Noman M, Van Assche G, Baert F, D’Haens G, Rutgeerts P. Effectiveness of concomitant immunosuppressive therapy in suppressing the formation of antibodies to infliximab in Crohn's disease. Gut 2007;56:1226-1231.

37. Su CG, Lichtenstein GR. Influence of immunogenicity on the long-term efficacy of infliximab in Crohn's disease. Gastroenterology 2003;125:1544-1546.

38. Niedziela JT, Hudzik B, Szygula-Jurkiewicz B, et al. Albumin-to-globulin ratio as an independent predictor of mortality in chronic heart failure. Biomark Med 2018;12:749757.
39. Zhou T, Yu ST, Chen WZ, Xie R, Yu JC. Pretreatment albumin globulin ratio has a superior prognostic value in laryngeal squamous cell carcinoma patients: a comparison study. J Cancer 2019;10:594-601.

40. Yue W, Liu B, Gao L, et al. The pretreatment albumin to globulin ratio as a significant predictor in patients with diffuse large B cell lymphoma. Clin Chim Acta 2018;485:316322.

41. Lv GY, An L, Sun XD, Hu YL, Sun DW. Pretreatment albumin to globulin ratio can serve as a prognostic marker in human cancers: a meta-analysis. Clin Chim Acta 2018;476:8191.

42. Du XJ, Tang LL, Mao YP, et al. The pretreatment albumin to globulin ratio has predictive value for long-term mortality in nasopharyngeal carcinoma. PLoS One 2014;9:e94473.

43. Liu Z, Huang H, Li S, et al. The prognostic value of preoperative serum albumin-globulin ratio for high-grade bladder urothelial carcinoma treated with radical cystectomy: a propensity score-matched analysis. J Cancer Res Ther 2017;13:837-843.

44. Li J, Wang Y, Wu Y, Li J, Che G. Prognostic value of pretreatment albumin to globulin ratio in lung cancer: a metaanalysis. Nutr Cancer 2021;73:75-82.

45. Dipasquale V, Mattioli G, Arrigo S, et al. Pouchitis in pediatric ulcerative colitis: a multicenter study on behalf of Italian Society of Pediatric Gastroenterology, Hepatology and Nutrition. Dig Liver Dis 2019;51:1551-1556.

46. Diakos CI, Charles KA, McMillan DC, Clarke SJ. Cancerrelated inflammation and treatment effectiveness. Lancet Oncol 2014;15:e493-e503 